AGráría

Revista Brasileira de Ciências Agrárias

v.4, n.1, p.17-21, jan.-mar., 2009

Recife, PE, UFRPE, www.agraria.ufrpe.br

Protocolo 332 - 23/02/2006 - Aprovado em 24/10/2008

\author{
Alberto S. de Melo ${ }^{1}$ \\ Marcos E. B. Brito² \\ Jolly D. de M. Dantas ${ }^{3}$ \\ Carlos D. da Silva Júnior 4 \\ Pedro D. Fernandes 5 \\ Lilianni V. Bonfim ${ }^{6}$
}

${ }^{1}$ Prof. Dr. Departamento de Agrárias e Exatas - DAE - Universidade Estadual da Paraíba - UEPB/ Campus IV. CEP:58.884-000, Catolé do Rocha PB.E-mail: alberto@uepb.edu.br. Autor para correspondência

${ }^{2}$ Engenheiro Agrônomo, MSc. Doutorando em Engenharia Agrícola - Universidade Federal de Campina Grande - UFCG. CEP: 58109-010, Campina Grande - PBE-mail: mebbrito@yahoo.com.br

${ }^{3}$ Engenheira Agrônoma, Mestranda em Agroecossistemas - Departamento de Engenharia Agronômica - DEA - Universidade Federal de Sergipe - UFS. CEP: 49.100-000, São Cristóvão SE. E-mail: jollydayanne@yahoo.com.br ${ }^{4}$ Prof. Dr. Departamento de Biologia - DBI- UFS. Email: cdias@ufs.br

${ }^{5}$ Prof. Dr. DEAg - UFCG-Campina Grande - PB. Email: pdantas@ufcg.edu.br

${ }^{6}$ Graduanda em Engenharia Agronômica - DEA UFS. E-mail: lilalivb@hotmail.com

\section{Produção e qualidade do pimentão amarelo sob níveis de potássio em ambiente protegido}

\section{RESU MO}

Realizou-se um experimento em casa de vegetação com objetivo de avaliar o efeito do suprimento de potássio na produção e na qualidade de frutos de pimentão amarelo (híbrido Zarco). 0 delineamento experimental foi o inteiramente casualizado, constituído por cinco níveis de potássio (1: 0,0 g

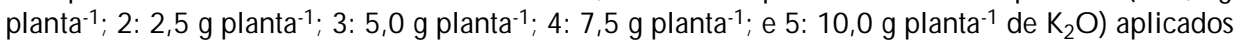
via fertirrigação, com quatro repetições, totalizando 20 parcelas, sendo cada parcela composta por três plantas úteis, dispostas em vasos de 10L de volume. Aos 90 dias após o transplante as plantas foram cortadas e avaliadas em relação ao peso de frutos por planta (PFP), número de frutos por planta (NFP), peso de frutos por classe (PFC) A, B e C, percentual de frutos por classe A, B e C e espessura de casca (ESPC) (mm). 0 fornecimento da dose de $7,0 \mathrm{~g}$ de $\mathrm{K}_{2} \mathrm{O}$ planta-1 promoveu a melhoria da qualidade da fruta do pimentão amarelo de acordo com os resultados da classificação do comprimento e do diâmetro do fruto. Recomenda-se a dose $10 \mathrm{~g} \mathrm{planta}^{-1}$ de $_{2} \mathrm{O}$ para melhoria da produção do pimentão cultivado em ambiente protegido.

Palavras-chave: Capsicum annuum, nutrição mineral, fertirrigação

\section{Yellow pepper yield and quality under potassium levels in a greenhouse}

\section{ABSTRACT}

An experiment was carried out in a greenhouse to evaluate the effect of potassium supply on the production and quality of yellow pepper fruits (hybrid Zarco). A completely randomized experimental design was used, with five potassium levels ( $1: 0.0 \mathrm{~g} \mathrm{plant}^{-1} ; 2: 2.5 \mathrm{~g} \mathrm{plant}^{-1} ; 3: 5.0 \mathrm{~g} \mathrm{plant}^{-1} ; 4: 7.5$ g plant ${ }^{-1}$; and 5: 10.0 g plant $^{-1}$ of $\mathrm{K}_{2} \mathrm{O}$ ) applied via fertigation, and four replicates, totaling 20 plots. Each plot was formed by three plants, disposed in $10 \mathrm{~L}$ volume pots. At 90 days after transplanting, the experiment was harvested and the following characteristics were evaluated: fruit weight per plant (PFP), number of fruits per plant (NFP) and fruit w eight per class (PFC) A, B and C, fruit percentage per class $A, B$ and $C$ and peel thickness (ESPC) (mm). The application of $7.0 \mathrm{~g}$ of $\mathrm{K}_{2} \mathrm{O}$ plant ${ }^{-1}$ provided the best quality of yellow pepper fruits, according to results of length and diameter classification. The application of $10 \mathrm{~g} \mathrm{plant}^{-1}$ of $\mathrm{K}_{2} \mathrm{O}$ is recommended to improve production of the yellow pepper cultivated under greenhouse conditions.

Key words: Capsicum annuum, mineral nutrition, fertigation 


\section{INTRODUÇÃO}

No agronegócio brasileiro, a produção de hortaliças apresenta alto potencial em virtude de sua importância nos setores sócio-econômico e alimentar. Estima-se que a produção de hortaliças agregue em torno de US\$ 9.750 milhões, o equivalente a 3,5\% do PIB agrícola, merecendo destaque na geração de empregos diretos (AGRIANUAL, 2004). Dentre as espécies hortícolas, o pimentão (Capsicum annuum) destaca-se com um volume comercializado no CEAGESP/SP de $37.686 \mathrm{t}$ durante o ano de 2003 (AGRIANUAL, 2004), situando-se entre as dez hortaliças mais importantes no Brasil, em termos de valor econômico.

No estado de Sergipe, o pimentão é uma cultura com grande potencial, visto que já é cultivado nos perímetros irrigados do Agreste sergipano, Jacarecica II e Poção da Ribeira e no alto Sertão, nos perímetros irrigados Califórnia e JacaréCurituba, gerando rendas e divisas (SEAGRI-SE, 2001). Relata-se, ainda, o crescimento da produção em ambiente protegido, o que permite escalonamento da produção e melhoria da qualidade dos frutos, em razão do maior controle das condições de cultivo. Com isso diminui-se a incidência de pragas e doenças, obtém-se proteção contra chuvas indesejáveis e reduz-se o consumo de água por unidade de produto agrícola produzido (Stanghellini, 1993).

Silva (2002) observou que, no cultivo de pimentão em ambiente protegido sob salinidade, o acúmulo de sais no solo em ambiente protegido é relacionado ao aumento da concentração de sais na solução. Ressalta-se que estes sais podem ter origem da água de irrigação de qualidade inferior, assim como nos fertilizantes aplicados, o que pode ser controlado com uso eficiente da fertilização aplicando a dose recomendada no momento, ou seja, de maior demanda da cultura.

Para o desenvolvimento satisfatório e atendimento das necessidades nutricionais e hídricas das plantas, notadamente em ambiente protegido, é essencial o uso de sistemas de irrigação que possibilitem maior freqüência de irrigação, bem como o uso da fertirrigação. Segundo Nannetti et al. (2000), a fertirrigação melhora a aplicação de nutrientes, aproximandose da marcha de absorção da planta, o que se torna ainda mais importante no estado de Sergipe, que possui solos de baixa fertilidade natural.

Epstein \& Bloom (2006) relatam que o potássio e o nitrogênio são os nutrientes mais exportados pelas plantas de pimentão, de modo que doses altas são aplicadas em cobertura para melhorar a eficiência da adubação diminuindo perdas por percolação e volatilização. Silva et al. (2001a) destacaram a importância do potássio no metabolismo do pimentão, que melhora as características físicas e químicas dos frutos, em razão da otimização no processo de transpiração e formação de carboidratos (Taiz \& Zeiger, 2004). No entanto, ao ser aplicado excessivamente, pode ocasionar distúrbios fisiológicos como queda na produção e qualidade dos frutos, desbalanço nutricional e aumento da pressão osmótica (Marschner, 1995). Porém, sua deficiência pode reduzir o rendimento e a qualidade dos frutos (Silva et al., 2001b).

Caso não haja uma recomendação de adubação via fertirrigação com potássio para o cultivo do pimentão amarelo em ambiente protegido, torna-se necessário o estudo dessa técnica de forma a proporcionar melhoria na qualidade dos frutos e, por fim, controlar eventuais distúrbios fisiológicos, sobretudo para as condições edafoclimáticas do Nordeste brasileiro. Assim, objetivou-se avaliar o suprimento de potássio via fertirrigação na produção e qualidade de frutos de pimentão híbrido amarelo cv. Zarco.

\section{MATERIAL E MÉTODOS}

O experimento foi realizado em casa de vegetação do Departamento de Biologia da Universidade Federal de Sergipe - UFS, localizado no município de São Cristóvão - SE.

O delineamento experimental foi inteiramente casualizado, constituído por cinco níveis de potássio (1: $0,0 \mathrm{~g} \mathrm{planta}^{-1} ; 2$ : 2,5 g planta $^{-1}$; 3: 5,0 g planta ${ }^{-1}$; 4: 7,5 g planta $^{-1}$; e 5: 10,0 g planta $^{-1}$ de $\mathrm{K}_{2} \mathrm{O}$, na forma de cloreto de potássio) em quatro repetições, totalizando 20 parcelas. A parcela foi composta por três plantas úteis de pimentão amarelo, híbrido Zarco, cada uma plantada em um vaso de $10 \mathrm{~L}$ preenchido com solo retirado da camada de 0-20 cm de um solo classificado como Argissolo Vermelho Amarelo Distrófico (EMBRAPA, 1999). Este solo apresenta textura franco-arenosa, com as seguintes características químicas: $\mathrm{pH}$ em água $=5,4$; matéria orgânica $=1,84 \mathrm{dag} \mathrm{kg}^{-1} ; \mathrm{Ca}^{2+}=9,23, \mathrm{Mg}^{2+}=5,95 \mathrm{e} \mathrm{K}^{+}=0,22 \mathrm{cmol}_{\mathrm{c}} \mathrm{kg}^{-}$ 1 .

No enchimento, cada vaso recebeu uma adubação de fundação de $400 \mathrm{mg}$ de superfosfato simples (18\% de $\left.\mathrm{P}_{2} \mathrm{O}_{5}\right)$ e 100 mg de FTE-BR12 como fonte de micronutrientes, bem como a aplicação de $100 \mathrm{~g}$ de calcário dolomítico para a correção de $\mathrm{Ca}$ e Mg (Raij et al., 1996). Os fertilizantes foram misturados a $10 \mathrm{~L}$ de solo, de modo que em $2 \mathrm{~L}$ foram misturados $50 \%$ do calcário e acondicionados na parte inferior do vaso, com vistas ao fornecimento de cálcio às plantas quando o sistema radicular atingir maior profundidade. Ao restante do volume foram adicionados todos os adubos, porém no terço superior foi misturado o FTE-BR 12, a fim de melhorar a disponibilização de micronutrientes.

Durante o experimento foram realizados os tratamentos fitossanitários por intermédio de aplicações semanais de inseticidas, para o controle de afídios, e de fungicidas preventivos á base de oxicloreto de cobre. As plantas foram cultivadas com três ramos, retirando-se os primeiros frutos de cada ramo para possibilitar melhor fluxo de seiva e padronização dos frutos (Santana et al., 2004; Fontes et al., 2005).

As fertirrigações foram efetuadas semanalmente, nas quais as doses de potássio foram adicionadas conforme os tratamentos. Foram adicionados a cada fertirrigação 1,5 g de adubo nitrogenado na forma de uréia (45\% de N) (Raij et al., 1996) e o potássio correspondente a cada tratamento.

A partir dos 90 dias após o transplante, os frutos com pelo menos $50 \%$ de sua coloração amarela foram coletados, pesados e padronizados em classes comerciais e não-comerciais (Frutos tipo A: maior que $100 \mathrm{~g}$; B: entre 50 e $100 \mathrm{~g}$; e C: menor que 50 g) (Damatto Júnior et al., 2005; Fontes et al., 2005; CEAGESP, 2008). As variáveis analisadas foram: diâmetro do fruto $(\mathrm{cm})$, comprimento do fruto $(\mathrm{cm})$, peso de frutos por 
planta (PFP), número de frutos por planta (NFP), peso de frutos por classe (PFC), percentual de frutos por classe e espessura de casca (ESPC) (mm).

Os resultados foram submetidos a ANAVA, teste $\mathrm{F}(\mathrm{p}<0,05)$, e os modelos de regressão polinomial, ajustados conforme o coeficiente de determinação pelo teste t (Student) até $5 \%$ de probabilidade de erro.

\section{RESULTADOS E DISCUSSÃo}

Com relação à classificação de frutos A (maior que $100 \mathrm{~g}$ ), $\mathrm{B}$ (entre 50 e $100 \mathrm{~g}$ ) e C (menor que $50 \mathrm{~g}$ ), observou-se aumento da proporção de frutos das classes A e B e diminuição dos frutos da classe $\mathrm{C}$, com o fornecimento de potássio no solo via fertirrigação (Figura 1). Constataram-se maiores valores médios das doses de $\mathrm{K}_{2} \mathrm{O}$ entre 6,6 e 8,8 g por planta, respectivamente, para frutos tipo A e B. Com a aplicação de doses de potássio acima de $8,8 \mathrm{~g}$ por planta, houve perda na qualidade. Silva et al. (2001b) reportaram que o aumento do teor de $\mathrm{K}_{2} \mathrm{O}$ no solo reduz a qualidade e a produção dos frutos. Este resultado decorreu possivelmente dos efeitos antagônicos provocados pelo excesso de $\mathrm{K}^{+}$no solo, que afeta a absorção de $\mathrm{Ca}^{2+}$ e $\mathrm{Mg}^{2+}$ pelos vegetais (Epstein \& Bloom, 2006). Ressalta-se que o Ca está envolvido na estrutura da lamela média e da parede celular, o que lhes confere qualidade e valor agrícola do produto.

Observaram-se maiores valores médios de diâmetro e comprimento dos frutos nas doses entre 6,45 e $6,98 \mathrm{~g}$ de $\mathrm{K}_{2} \mathrm{O}$ por planta, obtendo-se $6,25 \mathrm{~cm}$ de diâmetro e $9,98 \mathrm{~cm}$ de comprimento do fruto, o que realça a importância da aplicação correta de fertilizantes, a fim de maximizar o sistema de produção e aumentar a eficiência no uso de recursos. Entretanto, Marcussi et al. (2004), trabalhando com doses de nitrogênio e potássio via fertirrigação na cultura do pimentão, não observaram diferença significativa entre os tratamentos, assim como Nannetti et al. (2000). Portanto, os resultados obtidos neste trabalho diferem dos da literatura, pois verificou-se efeito das doses de potássio, em decorrência provavelmente das condições edafoclimáticas distintas entre os experimentos, bem como das exigências nutricionais de cada genótipo.

A maior espessura da casca $(0,5 \mathrm{~cm})$ foi obtida na dose de 9,0 $\mathrm{g} \mathrm{de} \mathrm{K}_{2} \mathrm{O}$ planta $^{-1}$. Moreno et al. (1996) e Scivittaro (1999), trabalhando com níveis de $\mathrm{N}$ e $\mathrm{K}$ em pimentão, relataram que o aumento de doses de $\mathrm{K}$ diminuiu a espessura de casca, o comprimento e o diâmetro dos pimentões, resultados diferentes dos observados neste trabalho. Ressalta-se que o potássio é importante para a translocação e alocação de açúcares da planta para os frutos, propiciando melhoria de suas características (Epstein \& Bloom, 2006).

Observou-se efeito dos níveis de potássio aplicados sobre o número de frutos por planta, peso de fruto por classe $(\mathrm{A}, \mathrm{B}$ e $\mathrm{C})$ e peso de fruto por planta $(\mathrm{p}<0,05)$ (Figura 2$)$.

O aumento dos níveis de potássio elevou o peso total de frutos acima de $100 \mathrm{~g}$ (Figura 2A) e entre 50 e $100 \mathrm{~g}$ (Figura 2B) e reduziu, em conseqüência, o de frutos abaixo de $50 \mathrm{~g}$ (Figura 2C), o que pode ser relacionado à melhoria na condutância estomática e no acúmulo de carboidratos decorrente da disponibilidade de potássio (Taiz \& Zeiger, 2004). Obtiveram-se maiores pesos e número de frutos por planta na dose de $10 \mathrm{~g} \mathrm{planta}^{-1}$ (Figuras 1D e 1E), valores abaixo daqueles relatados por Marcussi et al (2004), que constataram aumento linear da produção até a dose de 13,3 g por planta.

Moreno et al. (1996) constataram que, à medida que se aumentou o suprimento de $\mathrm{K}$ por planta em vasos, houve decréscimo na produção e incremento do teor de potássio no solo, promovendo o risco de salinização, o que pode explicar a redução na produção após o pico de máximo para estas
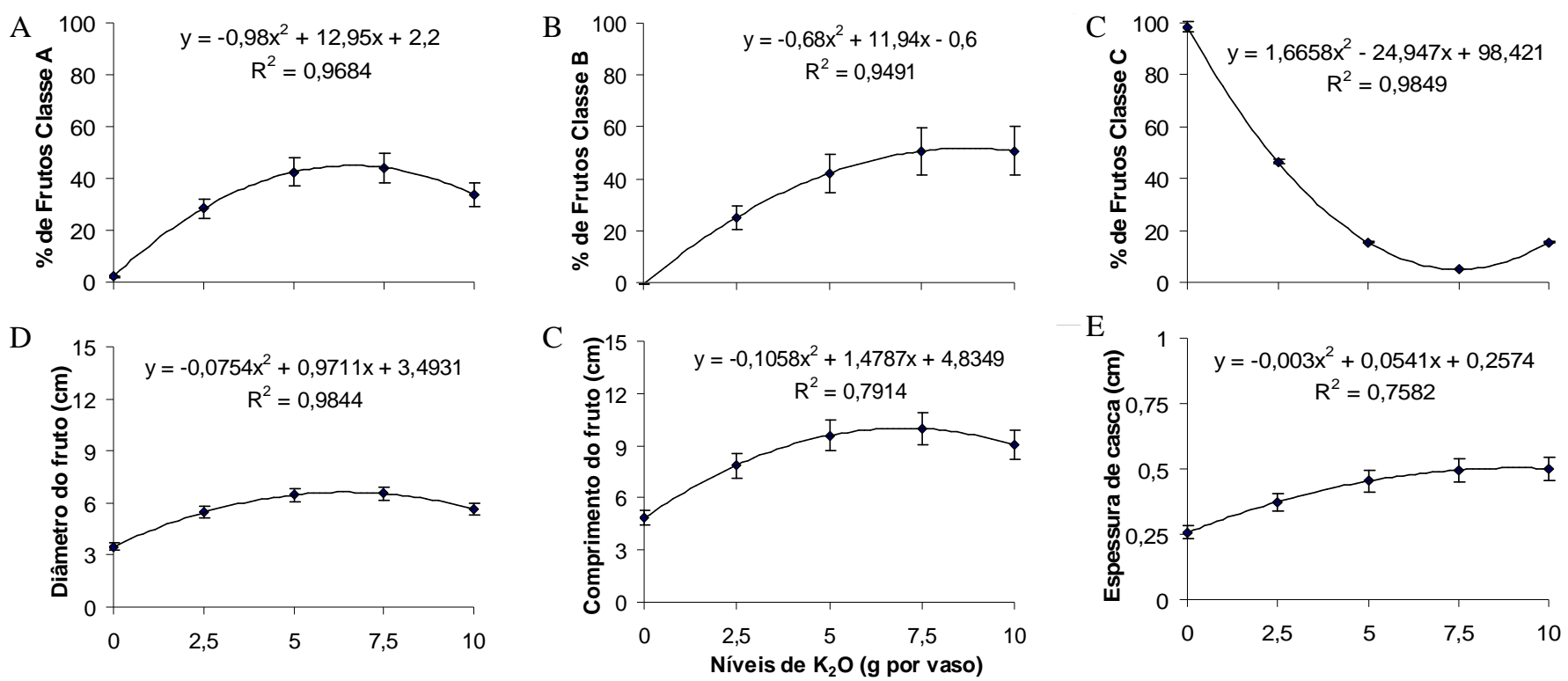

Figura 1. Porcentagem de frutos ( $A, B$ e C) $(A, B$ e C), diâmetros dos frutos (DF em cm) (D), comprimento do fruto (CF em cm) (E), e espessura de casca (ESPC em $\mathrm{cm})(\mathrm{F})$ em função das doses de potássio

Figure 1. Fruits percentage $(A, B$ and $C)$, fruit diameter $(D F$ in $\mathrm{cm})(D)$, fruit length $(\mathrm{cm})(E)$, and peel thickness $(E S P C$ in $\mathrm{cm})(F)$ as related to potassium rates 

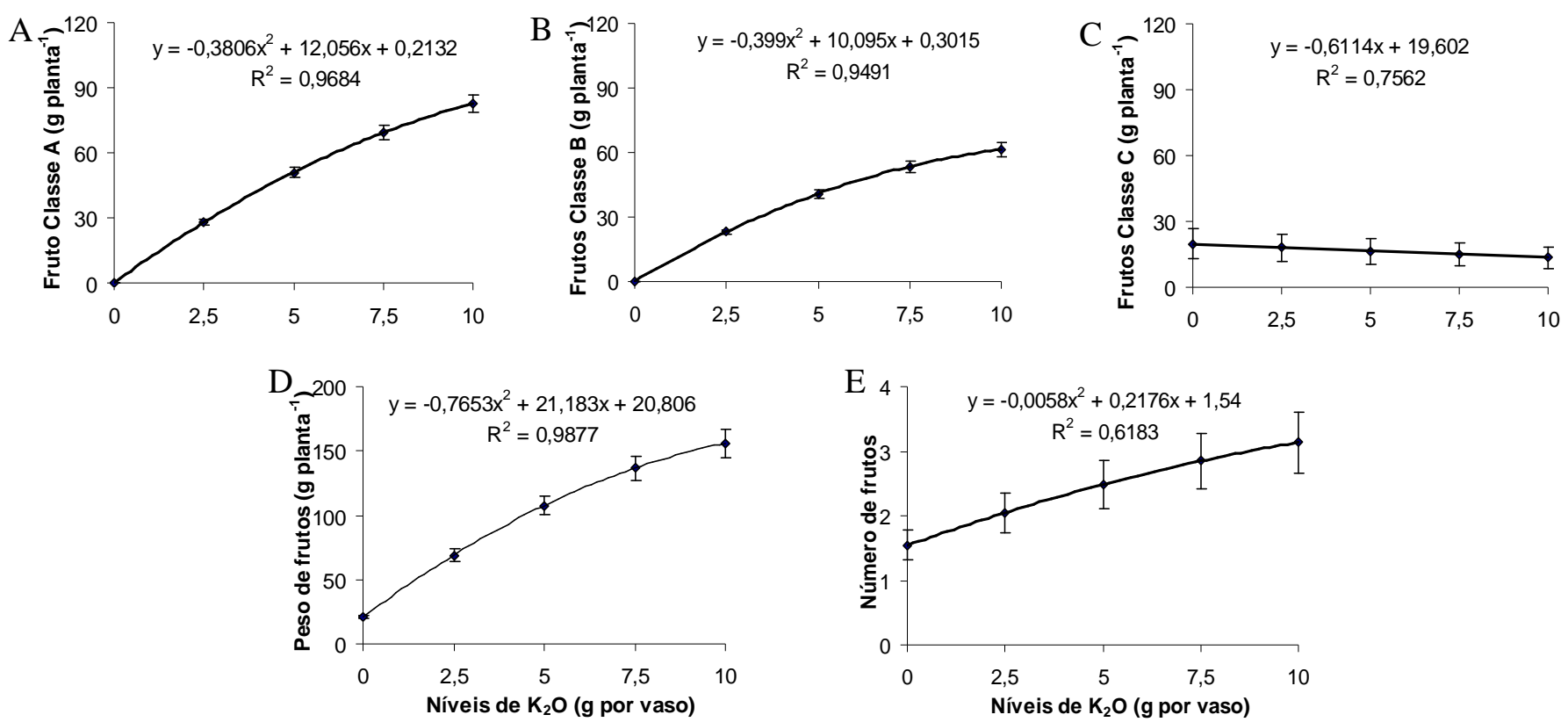

Figura 2. Comportamentos das variáveis, peso de frutos por classe(PFC) $(A, B$ e C) peso dos frutos por planta (PFP) (D) e número de frutos (E), em função das doses de potássio

Figure 2. Fruits weight for class (PFC) $(A, B$ and $C)$, fruits weigh for plant (PFP) $(D)$ and fruits number $(E)$, as related to potassium rates

variáveis neste trabalho. Isso mostra o efeito deletério do excesso de $\mathrm{K}$ no solo na produção de frutos de pimentão. Deve-se atentar também para a possível demanda de energia da planta para fazer o ajuste osmótico e absorção de água e nutrientes, o que poderia ser convertida em produção (Taiz $\&$ Zeiger, 2004). Os resultados deste trabalho não corroboram com os observados por Hassan \& Ramlan (1994), que constataram incremento linear no número e na produção de frutos com o aumento dos teores de potássio no solo. Salienta-se que a diversidade de resultados deve-se ao sistema de cultivo distinto. No contexto, para o alcance do máximo de produção, é necessária a dosagem correta respeitando a marcha de absorção do vegetal, ou seja, o atendimento da exigência nutricional em $\mathrm{K}$ pela cultura do pimentão, considerando o ciclo fenológico da planta.

\section{CONCLUSÕES}

$\mathrm{O}$ uso da dose de $7,0 \mathrm{~g}$ de $\mathrm{K}_{2} \mathrm{O}$ planta $^{-1}$ melhora a qualidade da fruta do pimentão amarelo, de acordo com os resultados da classificação: comprimento e diâmetro do fruto, em condições de solo de baixa fertilidade em K.

Recomenda-se a dose de 10 g planta $^{-1}$ de $\mathrm{K}_{2} \mathrm{O}$ para melhoria da produção do pimentão híbrido Zarco cultivado em casa de vegetação, quando o solo apresentar níveis baixos de $\mathrm{K}$.

\section{AGRADECIMENTOS}

À Empresa Syngenta Seeds®, pela doação das sementes de pimentão.

\section{LITERATURA CITADA}

Anuário da agricultura brasileira - AGRIANUAL. São Paulo: FNP Consultório e Comércio, 2004, 496p.

Companhia de Entrepostos e Armazéns Gerais de São Paulo CEAGESP. Padronização: pimentão. http:/ www.Ceasacampinas.com.br./padronização_pimentao.htm. 10 Dez. 2008.

Damatto Júnior, E.R.; Goto, R.; Vicentint, N.M.; Rodrigues, D.S.; Campos, A.J. Qualidade de frutos de pimentão amarelo em função de lâminas de água e cobertura do solo. Agronomia, v.29, n.1-2, p.49-54, 2005.

Empresa Brasileira de Pesquisa Agropecuária - EMBRAPA. Serviço Nacional de Levantamento e Conservação de Solos. Manual de métodos de análises de solo. Rio de Janeiro: EMBRAPA, 1999.73p.

Epstein, E., Bloom, A.J. Nutrição mineral de plantas: princípios e perspectivas. 2.ed. Trad.. Londrina: Editora Planta, 2006. 392p.

Fontes, P.C.R.; Dias, E.N.; Silva, D.J.H. Dinâmica do crescimento, distribuição de matéria seca e produção de pimentão em ambiente protegido. Horticultura Brasileira, Brasília, v.23, n.1, p.94-99, 2005.

Hassan S.A.; Ramlan, Z. A. Influence of potassium fertilizer and mulching on growth and yield of chilli (Capsicum annuит L.). Acta Horticulture, n.369, p.311-318, 1994.

Marcussi, F.F.N.; Godoy, L.J.G. de; Villas Bôas, R.L. Fertirrigação nitrogenada e potássica na cultura do pimentão baseada no acumulo de $\mathrm{N}$ e K pela planta. Irriga, v.9, n.1, p.4151, 2004.

Marschner, H. Mineral nutrition of higher plants. Academisc Press, 1995. 889p. 
Medeiros, J.F. Manejo de água de irrigação salina em estufa cultivada com pimentão. Piracicaba: Escola Superior de Agricultura "Luiz de Queiroz"/USP 1998.152 p. Tese Doutorado.

Moreno, D.A.; Pulgar, G.; Víllora, G.; Romero, L. Effect of N and $\mathrm{K}$ on fruit production and leaf levels of $\mathrm{Fe}, \mathrm{Mn}, \mathrm{Zn}, \mathrm{Cu}$ and $\mathrm{B}$ and their biochemical indicator in capsicum plants. Phyton, v.59, n.1-2, p.1-12, 1996.

Nannetti, D.C.; Souza, R.J.; Faquin, V. Efeito da aplicação de nitrogênio e potássio, via fertirrigação, na cultura do pimentão. Horticultura Brasileira, v.18, p.843-844, 2000.

Raij, B. van.; Cantarella, H.; Quaggio, J.A.; Furlani, A.M.C. Recomendação de adubação e calagem para o Estado de São Paulo. Campinas: IAC, 1996. 285p.

Santana, M.J.; Carvalho, J.A.; Faquim, V.; Queiroz, T.M. Produção do pimentão (Capsicum annuum L.) irrigado sob diferentes tensões de água no solo e doses de cálcio. Ciência e Agrotecnologia, v.28, n.6, p.1385-1391, 2004.

Scivittaro, W.B. Rendimento e qualidade de frutos de pimentão cultivado em ambiente protegido em função do nitrogênio e potássio aplicados em cobertura. Scientia Agrícola, v.56, n.4, p.1199-1207. 1999.
Secretaria da Agricultura do Estado de Sergipe - SEAGRI.. Ações prioritárias para o desenvolvimento da fruticultura em Sergipe. Aracajú: SEAGRI, 2001. (CD-ROM).

Silva, E.F.F. Manejo de fertirrigação e controle da salinidade na cultura do pimentão utilizando extratores de solução do solo. Piracicaba: Escola Superior de Agricultura "Luiz de Queiroz"/USP, 2002. 136p. Tese Doutorado.

Silva, M.A.G.; Boaretto, A.E.; Fernandes, H.G.; Boaretto, R.M.; Melo, A.M.T.; Scivittaro, W.B. Características químicas de um latossolo adubado com uréia e cloreto de potássio em ambiente protegido. Scientia Agrícola, v.58, n.3, p.561-566, 2001a.

Silva, M.A.G; Boaretto, R.M.; Fernandes, H.G.; Scivittaro, W.B. Efeito do cloreto de potássio na salinidade de um solo cultivado com pimentão, Capsicum annuиm L., em ambiente protegido. Acta Scientiarum.Agronomy, v.23, n.5, p.10851089, $2001 \mathrm{~b}$.

Stanghellini, C. Evapotranspiration in greenhouse with special reference to medirranean conditions. Acta Horticulturae, n.233, p.296-304, 1993.

Taiz, L.; Zeiger, E. Fisiologia vegetal. Porto Alegre: ArtMed, 2004. 719p. 